\title{
Stochastic hierarchical watershed cut based on disturbed topographical surface
}

\author{
Carlos Alberto F. Pimentel Filho ${ }^{\ddagger}$, Arnaldo de Albuquerque Araújo*, Jean Cousty ${ }^{\dagger}$, \\ Silvio Jamil F. Guimarães ${ }^{\ddagger}$, Laurent Najman ${ }^{\dagger}$ \\ †udio-Visual Information Proc. Lab. (VIPLAB) \\ Computer Science Department - ICEI - PUC Minas \\ fragapimentel@gmail.com sjamil@pucminas.br \\ *NPDI/DCC/UFMG \\ Federal University of Minas Gerais - Computer Science Department \\ Belo Horizonte, MG, Brazil \\ arnaldo@dcc.ufmg.br \\ ${ }^{\dagger}$ Université Paris-Est, Laboratoire d'Informatique Gaspard-Monge UMR 8049, \\ UPEMLV, ESIEE Paris, ENPC, CNRS, F-93162 Noisy-le-Grand France \\ \{j.cousty,l.najman\} @esiee.fr
}

\begin{abstract}
In this article we present a hierarchical stochastic image segmentation approach. This approach is based on a framework of edge-weighted graph for minimum spanning forest hierarchy. Image regions, that are represented by trees in a forest, can be merged according to a certain rule in order to create a single tree that represents segments hierarchically. In this article, we propose to add a uniform random noise into the edge-weighted graph and then we build the hierarchy with several realizations of independent segmentations. At the end, we combine all the hierarchical segmentations into a single one. As we show in this article, adding noise into the edge weights improves the segmentation precision of larger image regions and for F-Measure of objects and parts.
\end{abstract}

Keywords-watershed; stochastic segmentation; hierarchical segmentation; mathematical morphology.

\section{INTRODUCTION}

Image segmentation aims to obtain boundaries or frontiers of regions of interest from a given image. Thus, image segmentation plays a key role on image analysis as a fundamental step for several applications like image retrieval, classification [1], automatic annotation, and others. Although image segmentation has been researched for more than 40 years, it is still a challenging problem due to its complexity and the semantic nature inherent to this problem. Among several approaches that can be used for image segmentation, watershed transformation is one of the most powerful tools.

Considering a grayscale image as a topographic surface: the gray level of a pixel becomes the elevation of a point, the basins and valleys of the topographic surface correspond to dark areas, whereas the mountains and crest lines correspond to the light areas. Intuitively, the watershed divide is a set of points which satisfy the "drop of water principle": a separating set of points from which a drop of water can flow down towards at least two regional minima.

Aiming to compute the watershed of a digital image, several approaches have been proposed [2]-[7]. Many of them consider a grayscale digital image as a vertex-weighted graph. One of the most popular consists of simulating a flooding of the topographic surface from its regional minima [3], [8]. The divide is made of "dams" built at those points where water coming from different minima would meet. Another approach, called topological watershed [6], [9], [10], allows the authors to rigorously define the notion of a watershed in a discrete space and to prove important properties not guaranteed by most watershed algorithms [11]. It consists of lowering the values of a map (e.g., the grayscale image) while preserving some topological properties, namely, the number of connected components of each lower cross-section. In this case, the watershed divide is the set of points which are not in any regional minimum of the transformed map.

Because of inherent noise and other minor structures typically present on images, a great number of catchment basins embedded on the topography is created. Thereby, when the topographical surface has a large number of catchment basins, watershed transformation produces a large number of regions of interest. In order to reduce the large number of segments, markers can be used on regions of interest, a process known as seeded watershed.

Automatically setting the markers for seeded image segmentation is not a trivial task, specially for complex images. Another successful technique which makes use of the waterfall algorithms [12] is hierarchical image segmentation [13]. Rather than preventing oversegmentation, hierarchical segmentation computes the importance of segments given some criteria like catchment basin area, volume, depth or other. Roughly, this technique merges catchment basins that belong to almost homogeneous image regions. Hierarchical image segmentation provides a way for analyzing images from a rough representation of the segments to a detailed description of their smaller parts.

This article is focused on hierarchical stochastic image seg- 
mentation of watershed cuts in an approach where the image topography receives a uniform distributed random noise, producing $n$ realizations of slightly different topographic surfaces for the watershed. Each noised topographical surface is then processed by the hierarchical watershed algorithm proposed by Cousty et al. [15], generating each one a hierarchical segmentation. At the end of the process, the $n$ hierarchical segmentation versions are then combined into a single one in order to generate the final hierarchical result. As far as we know, this is the first hierarchical stochastic watershed segmentation work. Concerning to stochastic watershed segmentation, most references present a few quantitative results, in this sense, another important contribution of this article is to evaluate a stochastic watershed segmentation using a database for segmentation evaluation (BSDS500 [16]).

The present work is structured as follows. Section II presents a review on stochastic watershed image segmentation with some highlighted works. Section III describes our proposal for hierarchical stochastic image segmentation based on noised topographical surfaces that are modeled with edgeweighted graphs. The section describes how noise is inserted on the edge weights and some improvements taken to enhance the results. Our experiments are presented in Section IV and Section $\mathrm{V}$ concludes this article.

\section{RELATED WORKS}

Stochastic watershed is an unsupervised seeded watershed segmentation approach introduced by Angulo et al. [17]. The main idea of the approach is to apply several realizations of random markers to build a Probability Density Function (PDF) of the most significant regions which is then segmented by volumetric watershed. Roughly, a series of $n$ realizations with $r$ random markers are generated for a watershed segmentation applied to color gradient. After obtaining several segmentation versions of random seeds, the process estimates the probability Density function $p d f(x)$ that is obtained from all contours by Parzen window method. Despite the use of random markers is obviously not an appropriate choice of seeds, this arbitrary choice will be balanced by the use of a given number of $n$ realizations, hence, filtering out non significant fluctuations. To avoid oversegmentation of the large watershed areas caused by uniform distribution of the markers, the authors proposed to partition the $p d f(x)$ of contours with the volume-based watershed in order to obtain the $r$ most significant regions. Thus, each catchment basin volume corresponds to its probability to be a region of segmentation. According to Angulo et al. [17], this approach overcomes the standard watershed algorithms when the objective is to segment complex images into a few number of regions or segments. The stochastic watershed proposed in [17] works better for images with homogeneous regions size, what implies on some limitations for the approach because image regions may have a wide variety of sizes in real images.

A similar work to [17], also based on estimation of Probability Density Function for stochastic watershed segmentation for hyperspectral images was proposed by Angulo et al. [18]. Besides the application of hyperspectral images, this work also presented an innovation according to the use of multiscale approach for the computation of the $p d f(x)$. In fact, two multiscacles approaches were presented, one linear scale-space using Gaussian filters and another, nonlinear, for morphological scale-space pyramid using levelings.

Aiming to avoid the split of large regions caused by uniform distribution of markers using Poisson process, the work presented by Noyel et al. [19] proposed to place markers with help of spectral classification of multispectral images. The approach also computes the PDF by Parzen kernel method. A highlighted advantage of the approach is that it requires a single parameter, which is the number of classes within the image instead of the number of segments. Thinking on the number of classes instead of regions is a better strategy because some kinds of image tend to have similar number of classes to be segmented, but not the same number of regions.

Recently, Bernarder et al. [20] proposed two modifications for stochastic watershed [17] aiming to improve the image segmentation. The first improvement was the addition of uniform distributed random noise on the input image at every iteration of the seeded watershed. The second modification is focused on the use of a random placed grid to distribute the markers in order to obtain the PDF. According to authors, the addition of uniform distributed random noise brought major positive impact on the work, rather than the random placement of grid. Further, the proposed algorithm does not break up large regions and can better handle stochastic watershed on images with different sizes of segments, which makes the algorithm useful for a large set of segmentation problems. Also, the output is less sensitive to the number of markers.

Finally, in [21], Malmberg et al. presented an efficient method for computing the stochastic watershed PDF without performing any actual seeded watershed computations. Further, an alternative method to estimate contour strength without need of several random markers simulation was presented by Meyer et al. in [22].

Aiming to improve the watershed cut algorithm using area criteria for extinction values, and to evaluate the segmentation quality on a wide variety of real images by quantitative measurements, we propose here an algorithm for stochastic hierarchical watershed which is presented in next section.

\section{Stochastic Hierarchical Watershed}

Due to noise on acquisition, digitalization, compression, transmission and/or other origin, natural image present a large number of local minima on their gradient topography. Several methods have been proposed to solve this problem, such as: (i) the removal of local minima on a preprocessing step; (ii) region merging on a postprocessing step; (iii) or the use markers for each region to be delineated. Thereby, on this last idea, each marked region subistitute all the minima of the region by the marker [20].

Seeded watershed segmentation presents one problem at uniform areas of the image. If two markers are inserted on the same uniform region, the segmentation algorithm will produce 
two segments. Aiming to avoid this pattern, the work presented in [20] adds a uniform distributed random noise of small energy on the image pixel values. This noise must be strong enough to be mixed to the natural noise of the image, however not too strong to destroy image objects structure.

In this work, we add noise in a edge-weighted graph representing the topographical surface of the image in a method that we call Stochastic Hierarchical Watershed (SHW). The idea behind this approach is to modify the noise inherent to images in different versions of the topographic surface. This modification changes minima position and borders of catchment basins with small dynamics [23] magnitude. In order to achieve this desired characteristic, noise energy must be as strong as the inherent image noise but week enough to not destroy or corrupt important ridges on the topographic surface, once they represent relevant borders of segments.

From the topographical surface of the image, a set of $n$ hierarchical segmentations are built from disturbed weights of the graph edges. Each hierarchical segmentation is them regularized by a Gaussian convolution and finally combined at the end in a single saliency map as illustrated in Fig. 1.

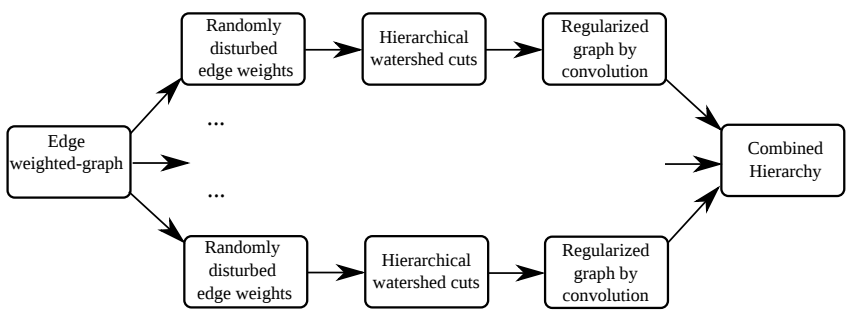

Fig. 1. Stochastic hierarchical watershed cut segmentation diagram.

\section{A. Random perturbation of edge weights}

The topographical surface of an image can be represented by a undirected edge-weighted graph where vertex represent pixels and edge weights represent the absolute difference between the values of two neighbor pixels. Adding noise to the graph edge weights, that in this work represents the topographical surface of an image, changes the configuration of the catchment basins with small ridges, nonetheless we still have an oversegmented image obtained by this process. To overcome this problem, it is necessary to obtain a set of segmentations provided by several versions of perturbed edge weights, each one obtained by the original image gradient modulus (topographical surface) softly modified by uniform random noise. In our proposal, the perturbation on the edge weights followed by the hierarchical image segmentation by watershed cuts is repeated $n$ times, creating $n$ independent saliency maps. At the end, the final result is obtained by an arithmetic average combination of those segmentations. In this work, instead of computing the PDF from seeded watershed, we use the approach of Cousty et al. [15] based on the hierarchical minimum spanning forests and saliency of watershed cuts to obtain hierarchical image segmentation, but in a stochastic method.

\section{B. Hierarchy of watershed cuts and saliency maps}

A hierarchical segmentation is a hierarchy of connected regions. Rather than preventing oversegmentation, it computes the importance of contours respecting some criteria. Let $\mathscr{P}_{h_{i}}$ be a set of partitions of a plane. The family $\left(\mathscr{P}_{h_{i}}\right)_{i}$ is a hierarchy if $h_{i} \geq h_{j}$ implies $\mathscr{P}_{h_{i}} \supseteq \mathscr{P}_{h_{j}}$, i.e., any region of partition $\mathscr{P}_{h_{i}}$ is a disjoint union of regions of partition $\mathscr{P}_{h_{j}}$ [13].

A useful representation of hierarchy, named saliency map, was first introduced by Najman et al. [13]. Every hierarchy can assigned to a saliency map, by evaluating each point on the plane by the highest value $h$ such that it appears in the boundaries of partition $\mathscr{P}_{h}$. Considering a partition equivalent to a segment, we can assign its importance to a contour.

According to [15], [24], [25], forests of minimum weight of the edge-weighted graph characterize watersheds associated with the set of minima, where each minima is rooted in a tree of the forest. The hierarchy of watershed cuts, produced by e.g. the algorithm [15], [26] are deeply linked to minumum spanning tree which preserves all the necessary information aiming to rank the minima according to the catchment basin mesurement criteria, either dynamics [23], either area, either volume or other one [15], [27], [28]. Those values to rank the catchment basins hierarchically are called extinction values [29]. For example, regarding to ranking criteria by area, the bigger is the basin area, the higher it must be placed in the hierarchy, while for dynamics, the deeper is the catchment basin, the higher this related segment is placed in the hierarchy.

\section{Edge-weighted graph convolution}

One observed characteristic on the SHW approach is that noise changes the configuration of catchment basins with small ridges, while do not affect big ridges or presents only a small disturbance but not destroying their structure. Those small ridges, usually present on small and uniform regions, appear in a lower position on the hierarchical segmentation, i.e., near to the leaves of the tree. By consequence, small ridges generate lower contrast boundary on the saliency map. In such way, each different version of saliency map provided by SHW drastically change contours position on lower hierarchical levels, while on higher hierarchical one, do not change or change just a little as observed in Fig. 2 (b), (c), (d) and in Fig. 4. In order to preserve contours at similar position and specially to weaken contours in disagreement position compared to different saliency maps of the same image, we experimented the use of a Gaussian convolution method directly applied to the edge-weighted graph representation of saliency map. We consider that relevant contours are repeated in the same or similar position, while contours in different positions are not that relevant. The convolution with a Gaussian kernel that we used can better preserve contours in the neighborhood, i.e., inside the kernel coverage, while it makes weaker the contours without repetition on its around. For that, we used the smallest possible kernel because we did not wanted to reinforce distant contours which may remain to different objects or segments. Three Gaussian kernel graphs for graph 


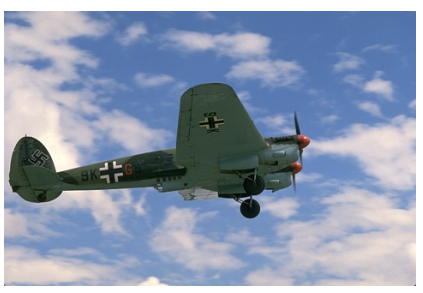

(a)

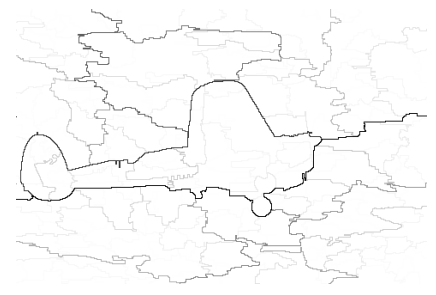

(b)

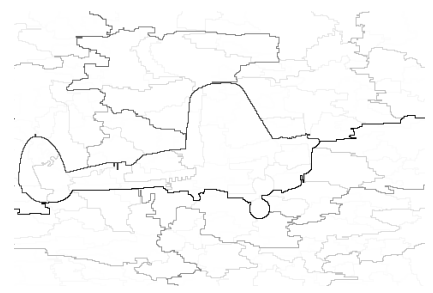

(c)

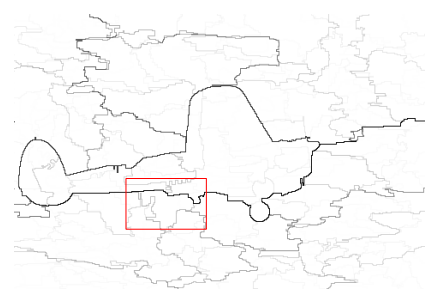

(d)

Fig. 2. (a): Original color image; (b): saliency map obtained from (a) with uniform random noise added on the topographic surface; (c): another example of saliency map obtained from (a); and (d) arithmetic average of saliency maps (b) and (c). Details on Fig. (d) show that while the strongest contours of (b) and (c) are preserved due to big ridges on the topographical surface, contours remaining to small catchment basins are slightly different due to the noise disturbance on the topographic surface of the image gradient obtained from (a).

convolution are presented in Fig. 3. In this figure, the three kernels $(K 1, K 2$ and $K 3)$ present small variation on their coverage and weights.

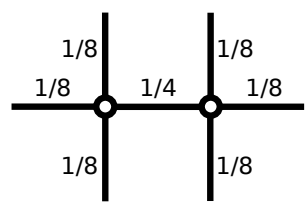

(K1)
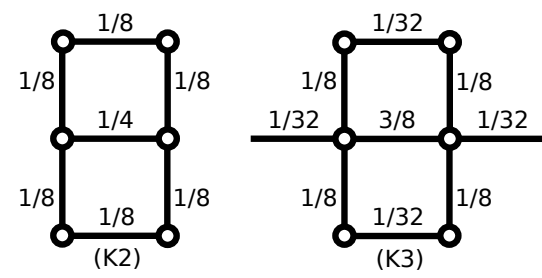

Fig. 3. Gaussian Kernel graphs used on the edge-weighted graph convolution. Edge weights are presented beside their edges.

Consider the segment of Fig. 2 (d) presented in detail in Fig. 4. This segment composed by two averaged saliency maps presents two examples detached respectively in the blue and the green dashed rectangles. On the blue one, the segment of the airplane object was slightly moved on each version of saliency map origin by the uniform noise. In this case, the position difference is very small and we want to keep this contour. The proposed kernels shown in Fig. 3 still cover the adjacent segment. Now consider the segment inside the green dashed rectangle. This segment, present only in one version of the original saliency map of Fig. 2 (b), is not important because appeared only once, thus it has no other border on its neighborhood and the Gaussian kernel will weaken it.

The graph convolution works in a similar way of image convolution, where graph edge weights substitute the pixel values.

The kernels presented on Fig. 3 are used for "horizontal" edges. The kernels used for "vertical" edges are isomorphic to kernels presented on Fig. 3, but they are rotated $90^{\circ}$.

\section{Stochastic hierarchy of watershed cuts}

The process of disturbing the edge-weighted graph is repeated $n$ times creating $n$ slightly modified topographic surfaces representations. Finally contours are combined at the end with arithmetic average. Let $G=(V, E)$ be an undirected weighted graph, where $V$ and $E$ are finite sets that represent respectively vertices and edges; $V \in\left\{v_{1}, v_{2}, \ldots, v_{k}\right\}$ and $E \in\left\{e_{1}, e_{2}, \ldots, e_{m}\right\}, k$ is the number of vertices and $m$ the

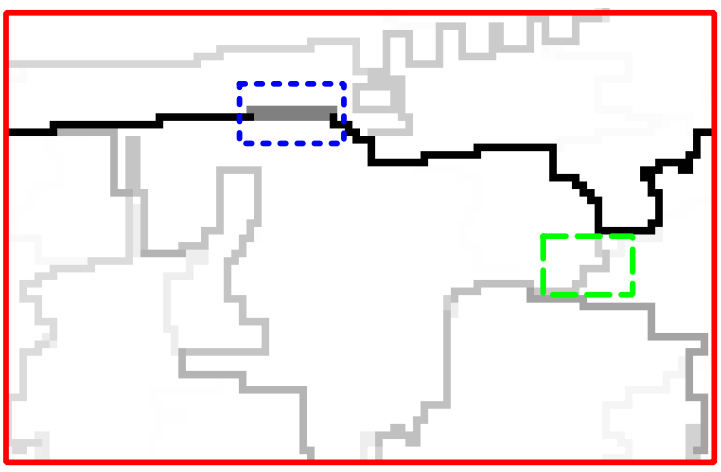

Fig. 4. Segment of Fig. 2 (d) detaching two cases of averaged contours of saliency maps of Fig. 2 (b) and (c). In the first case (blue dashed rectangle), two segments are softly misplaced by noise addition. In the second case (green dashed rectangle), one segment obtained only from saliency map of Fig. 2 (b).

number of edges. The arithmetic average of the edge-weights associated to a saliency map is obtained by Eq. 1:

$$
\mathscr{A}\left(w_{j}^{\prime}\right)=\frac{1}{n} \sum_{i=1}^{n} w_{j} \mid e_{j} \in E_{i}
$$

where $n$ represents the number of graphs in the set $\left\{G_{1}, G_{2}, \ldots, G_{n}\right\}$ and $w_{j}$ the weight associated to edge $e_{j}$.

At the end of this process, we obtain a hierarchical stochastic watershed segmentation using hierarchical minimum spanning forest and saliency watershed cuts as described in [15].

Fig. 2 illustrates some segmentation results of noise addition on the topographic surface of image in Fig. 2 (a). Figs 2 (b) and (c) represent two examples of saliency maps obtained by SHW of Fig 2 (a). Details on Figs 2 (b) and (c) reveal that slight differences on contours of both saliency maps are produced by the impact of noise on the topographic surface of the image. Figs 2 (d) presents an averaged saliency map obtained by the arithmetic average of two saliency maps (b) and (c).

\section{E. Stochastic topographical watershed algorithm}

The algorithm for the SHW approach is presented in Algorithm 1 . 


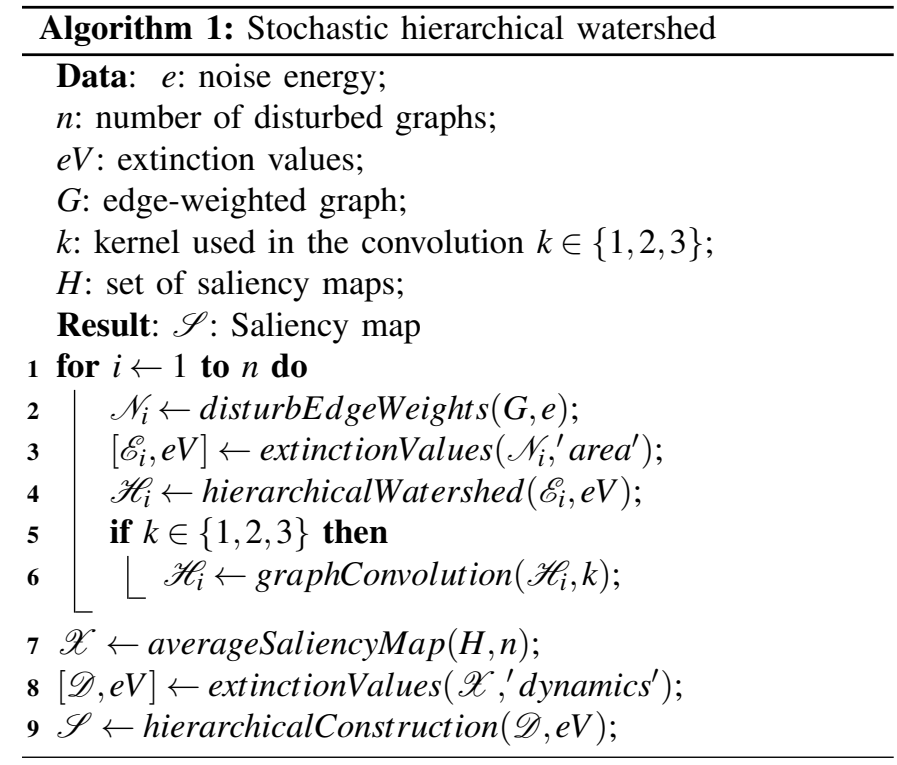

In this algorithm, given a undirected edge-weighted graph $G$; disturbEdgeWeights $(G, e)$ adds noise of intensity $e$ on the edge-weights of $G$ as described in Section III-A; extinctionValues $\left(\mathscr{N}_{i},{ }^{\prime}\right.$ criteria $\left.^{\prime}\right)$ determines the segment hierarchical order according to some criteria (area or dynimics); hierarchicalWatershed $\left(\mathscr{E}_{i}, e V\right)$ performs the hierarchical construction of segments of watershed cuts algorithm; graphConvolution $\left(\mathscr{H}_{i}, k\right)$ performs a graph convolution using one of the kernels presented in Fig. 3; and averageSaliencyMap $(H, n)$ computes the average weight for the edges of the set of graphs $H$ as presented in Eq. 1.

\section{EXPERIMENTS AND RESULTS}

In this section, we present a set of experimental results for Stochastic Hierarchical Watershed $(\mathrm{SHW})$ image segmentation. The experiments use the evaluation method ground-truth for covering described by Arbelaez et al. [14] and F-Measure for object and parts $F_{O P}$ described by Tuset et al. [30]. Details about the experiments are described on the following.

\section{A. Experimental setup}

In this work, we used RGB color images where each color channel is represented in discrete mode in the range $[0, \ldots, 255]$. We added random uniform noise in a 4-connected undirected edge-weighted graph representing the topographic surface of RGB gradient modulus [15]. Each edge weight represents the absolute difference between two neighbor pixels values sum of the three RGB chanels. This noise is random and uniformly distributed varying on the range $[-e, \ldots, 0, \ldots, e]$, where $e$ represents the noise energy defined on each experiment. After adding noise on the edge weights of the graph, negative values are truncated to 0 and values higher than 255 are truncated to 255. In order to assess our approach and evaluate its effectiveness, we used the image database of Martin et al. [16] (BSDS500) that consists in a set of 500 images and a groundtruth of human segmentations in a wide variety of natural scenes provided by Arbelaez [14]. We used 200 images with ground-truth from this database on each experimental method described on following.

The evaluation of image segments is performed using the approach comparison of covering. Roughly, according to Arbelaez [14], the segmentation of ground-truth covering is defined using as basis the overlap between two regions, $R$ and $R^{\prime}$. We say roughly because although the overlapping of regions is the basis of the covering measure, other minor elements are taken into consideration. For a detailed description of the ground-truth covering measure we recommend the reference [14]. Aiming to compare two different experiments, we used the paired t-test analysis considering the results of 200 images of each experiment.

\section{B. Stochastic hierarchical watershed (SHW) evaluation of parameters}

In this section, we present the results evaluating the parameters for stochastic hierarchical watershed $(\mathrm{SHW})$ using covering comparison. We experimented noise energy (Section IV-B1); the number of topographical noised surfaces (Section IV-B2), and convolution kernels (Section IV-B3). Finally, we compare the best result of our experiments with the non stochastic hierarchical watershed based on area (WArea) (Section IV-C).

1) Noise energy evaluation: The signal-to-noise ratio must be well established in such way that the noise inserted can be mixed to the inherent noise without destroying important image structures, i.e., without corrupting important basin ridges. This experiment evaluates this relation inserting a very low noise energy growing to higher magnitude. In Fig. 5 the bars respectively represents the experimental results of increasing noise energy $e=1,2,3,4$. We used three stochastic watershed saliency maps that are joined with arithmetic average $(\mathscr{A})$ and convolution was not used in the experiments of Fig. 5.

It is important to note that the data presented in Figs. 5, 6 and 7 correspond to the normal distribution of individual results on each image of the BSDS500 database. On the experiments, each image is evaluated 29 times, one for each ground-truth covering measurement considering from 2 until 30 segments. The best ground-truth covering of the interval [2-30] is taken to represent each image segmentation fitness on each experiment. Further, for Figs. 5, 6 and 7, we have: i) the median covering (central bar); ii) the first and third quartile (extremities of the box); iii) the lowest datum still within 1.5 inter quartile range (difference between the third and first quartile) of the lower quartile, and the highest datum still within 1.5 inter quartile range of the upper quartile range (left and right extremities); and $i v$ ) the outliers (individual points).

The lowest noise energy (Fig. 5 SHWel) presented the higher median value for covering. This result is also statistically better than higher energy ( $\mathrm{SHWe2}$, SHWe3 and SHWe4) as shown by the paired t-test analysis of Table I.

2) Number of saliency maps evaluation: An other important question raised for the hierarchical stochastic watershed was the best number of saliency maps that should be produced 


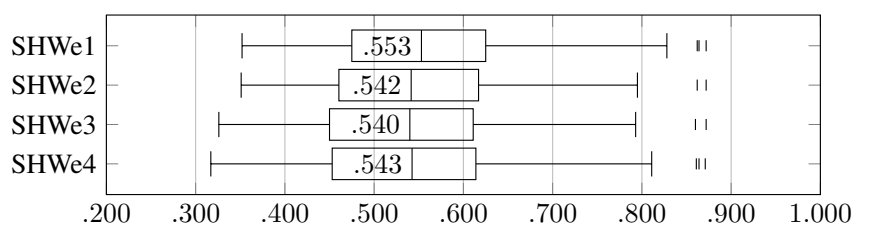

Fig. 5. Comparison for covering of stochastic hierarchical watershed with different levels of noise. From the first bar to the last one, we have increasing noise energy level. For the first bar; noise level $e=1$; for the second bar, $e=2$; for third bar $e=3$; and for the fourth bar $e=4$. On each experiment we used three stochastic watershed samples joined with arithmetic average. Convolution was not used in these experiments.

TABLE I

BEST METHOD CHOICE WITH CONFIDENCE INTERVAL FOR EACH PAIRWISE COMPARISON OF NOISE LEVEL.

\begin{tabular}{ccc}
\hline Methods & The best & Confidence interval (95\%) \\
\hline SHWe1 X SHWe2 & SHWe1 & {$[0.000864,0.018]$} \\
\hline SHWe1 X SHWe3 & SHWe1 & {$[0.00486,0.0225]$} \\
\hline SHWe1 X SHWe4 & SHWe1 & {$[0.00199,0.0195]$} \\
\hline SHWe2 X SHWe3 & equivalent & {$[-0.00232,0.0108]$} \\
\hline SHWe2 X SHWe4 & equivalent & {$[-0.00693,0.00946]$} \\
\hline SHWe3 X SHWe4 & equivalent & {$[-0.00977,0.00382]$} \\
\hline
\end{tabular}

to improve the segmentation accuracy. A small number of segmentations may not be enough while a large number may not be necessary. Aiming to evaluate this question we compared the use of several stochastic saliency maps in the set $n=\{3,6,9,25\}$, i.e., the average combination of three segmentations, six, nine and 25. Results are presented in Fig. 6, where the first bar represents the result of the experiment combining 3 saliency maps, the second bar 6 saliency maps, the third bar 9 saliency maps and the last bar 25 saliency maps.

In the experiment presented in Fig. 6, we used noise energy $e=1$ and arithmetic average $(\mathscr{A})$ of $n$ saliency maps. Convolution was not used. Note that $S H W n 3$ of Fig. 6 is equal to $S H W e 1$ presented in Fig. 5, once that those experiments present the same parameter setup.

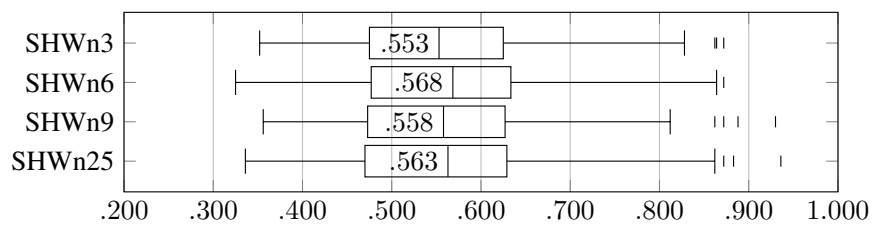

Fig. 6. Comparison of stochastic hierarchical watershed (SHW) with different number of saliency maps. From the first to the last bar, we have arithmetic averages respectively with $3,6,9$ and 25 different stochastic saliency maps.

Table II presents a paired t-test analysis of the experiments presented in Fig. 6. Although the results are all equivalent to one each other on the paired t-test, we chose $S H W n 6$ as the best because it presents a higher median value in Fig. 6.

3) Convolution kernels evaluation: The basic idea of adding noise to the image gradient, and at the end averaging the saliency maps, is that strong contours tend to be repeated
TABLE II

BEST METHOD CHOICE WITH CONFIDENCE INTERVAL FOR EACH PAIRWISE COMPARISON IN NUMBER OF SALIENCY MAPS.

\begin{tabular}{ccc}
\hline Methods & The best & Confidence interval (95\%) \\
\hline SHWn3 X SHWn6 & equivalent & {$[-0.0134,0.00219]$} \\
\hline SHWn3 X SHWn9 & equivalent & {$[-0.0105,0.00634]$} \\
\hline SHWn3 X SHWn25 & equivalent & {$[-0.0116,0.00398]$} \\
\hline SHWn6 X SHWn9 & equivalent & {$[-0.00244,0.00941]$} \\
\hline SHWn6 X SHWn25 & equivalent & {$[-0.004,0.0076]$} \\
\hline SHWn9 X SHWn25 & equivalent & {$[-0.00649,0.00313]$} \\
\hline
\end{tabular}

TABLE III

BEST METHOD CHOICE WITH CONFIDENCE INTERVAL FOR EACH PAIRWISE COMPARISON OF CONVOLUTION KERNELS.

\begin{tabular}{clc}
\hline \multicolumn{1}{c}{ Methods } & The best & Confidence interval (95\%) \\
\hline SHWn6 X SHWK1 & SHWK1 & {$[-0.0142,-0.000415]$} \\
\hline SHWn6 X SHWK2 & SHWn6 & {$[0.0109,0.0266]$} \\
\hline SHWn6 X SHWK3 & SHWn6 & {$[0.00586,0.0209]$} \\
\hline SHWK1 X SHWK2 & SHWK1 & {$[0.0181,0.0339]$} \\
\hline SHWK1 X SHWK3 & SHWK1 & {$[0.0138,0.0275]$} \\
\hline SHWK2 X SHWK3 & equivalent & {$[-0.0118,0.00109]$} \\
\hline
\end{tabular}

in the same position while noise disturbs weak contours that are not repeated in each version of saliency map. Further, weak noise can disturb the border position of strong contour, but not drastically. Aiming to weaken even more contours that appear in a completely different position, and to keep strong contours at near position, we apply a convolution with a Gaussian kernel. We experimented three different kernels with different coverage size and weights. The kernels are presented in Fig. 3. In Fig. 7, the first bar represents the experimental results of the $S H W$ without convolution while the following bars are experiments respectively with convolution Gaussian kernels $K 1, K 2$ and $K 3$. In the experiments shown in Fig. 7 we used six stochastic saliency maps and noise energy $e=1$.

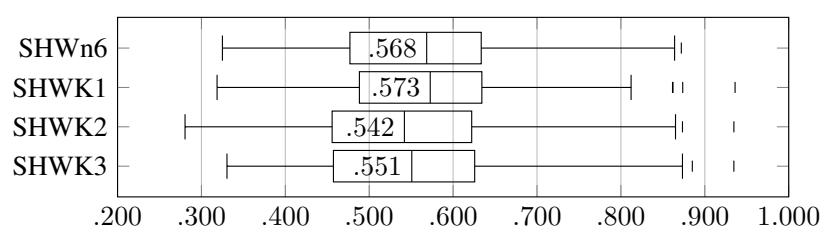

Fig. 7. Comparison for covering of stochastic hierarchical watershed without convolution and with convolution using three different kernels. First bar presents the experimental result of $S H W$ without convolution, the second bar presents the $S H W$ with convolution kernel $K 1$, the third bar convolution with kernel $K 2$ and the fourth bar convolution with kernel $K 3$. In this figure, all experiments used six stochastic saliency maps and noise energy $e=1$.

Table III presents a paired t-test analysis of one experiment without convolution and experiments using convolution with kernels $K 1, K 2$ and $K 3$. Following this analysis we conclude that the kernel $K 1$ presented the best result over the other kernels and the experiment without convolution. 
TABLE IV

BEST METHOD CHOICE WITH CONFIDENCE INTERVAL FOR PAIRWISE COMPARISON BETWEEN NON STOCHASTIC HIERARCHICAL WATERSHED BY AREA AND STOCHASTIC HIERARCHICAL WATERSHED BY AREA.

\begin{tabular}{ccc}
\hline Methods & The best & Confidence interval (95\%) \\
\hline WArea X SHWK1 & SHWK1 & {$[-0.0268,-0.0102]$} \\
\hline
\end{tabular}

\section{Comparing the stochastic and non stochastic approach based on area}

In this section we compare the standard hierarchical watershed by area (WArea) with the best result of stochastic hierarchical watershed $(S H W K 1)$. The stochastic method is the one combining the best parameters of each experiment presented, i.e., noise energy $e=1$, six stochastic saliency maps and convolution kernel $K 1$. Fig. 8 presents this comparison. First bar represents the standard hierarchical watershed area while the second presents the best result obtained in this work.

As shown in Fig. 8, the addition of uniform random noise on the image gradient outperforms the watershed cut algorithm using area criteria (WArea). Further paired t-test analysis presented in Table IV shows that method $S H W K 1$ is statistically better than WArea.

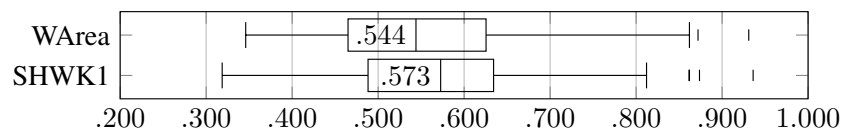

Fig. 8. Comparison of covering between hierarchical watershed area (WArea) and stochastic hierarchical watershed with six stochastic saliency maps, energy noise $e=1$ and convolution using kernel K1 (SHWK1).

1) Scale comparison with covering: In this section we present the results using the BSDS500 database with covering evaluation. However, instead of analyzing the results of individual images as presented in Section IV-B, the results presented here correspond to the whole database regarding to scale parameters. The scale parameter is related to the desired number of segments for each image of the database.

The scale parameter do not corresponds to the provided hierarchy, but it is somehow "normalized" to take into account only the 30 highest distinct provided segments. Thus, this experiment presents the average covering of segments from 200 images of each average between 2 and 30 segments.

Fig. 9 presents the results of covering comparison regarding to the number of segments. Area line (WArea) represents hierarchical watershed area. The other lines represent best results of each $S H W$ experiment: $S H W e 3$ from Fig. 5; SHWn6 from Fig. 6; and $S H W K 1$ from Fig. 7. Ordinate represents the covering measure while abscissa the number of segments from 2 to 30 .

Enforcing the covering results for single images presented in Section IV-B2, the covering results considering scale shows that stochastic hierarchical watershed overcomes the hierarchical watershed area. Although results of $S H W$ are considerable higher, especially for few number of segments, on the opposite

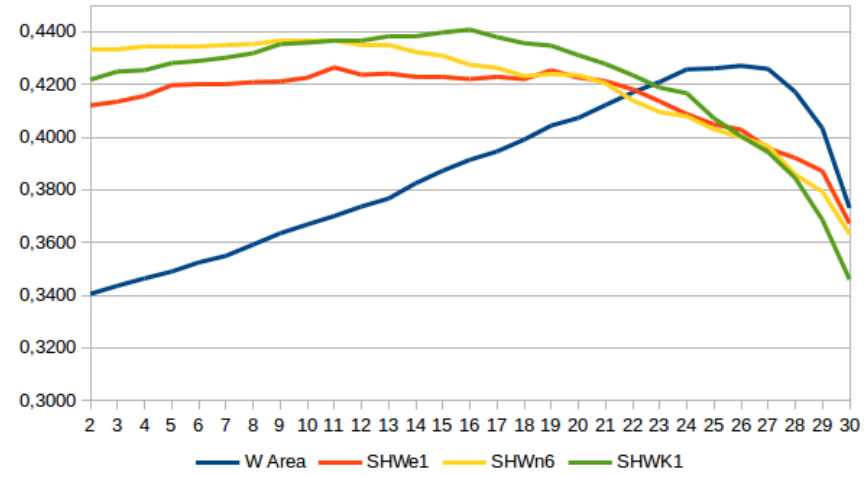

Fig. 9. Covering comparison between watershed area and stochastic watershed, from segment parameter 2 to 30 . Each line represents one experiment. The line WArea represents the baseline using watershed area cuts while the other lines represent the best results of $S H W$ on each experimental setup.

side of the scale parameter where we have 23 segments or more, non stochastic watershed area presents better results.

2) F-Measure for object and parts $\left(F_{O P}\right)$ comparison: In this section we present the experimental results using $F_{O P}(\mathrm{~F}-$ Measure for object and parts) [30]. Like on the experiments presented on Sections IV-C1, the experiments using $F_{O P}$ are evaluated for the desired number of parts.

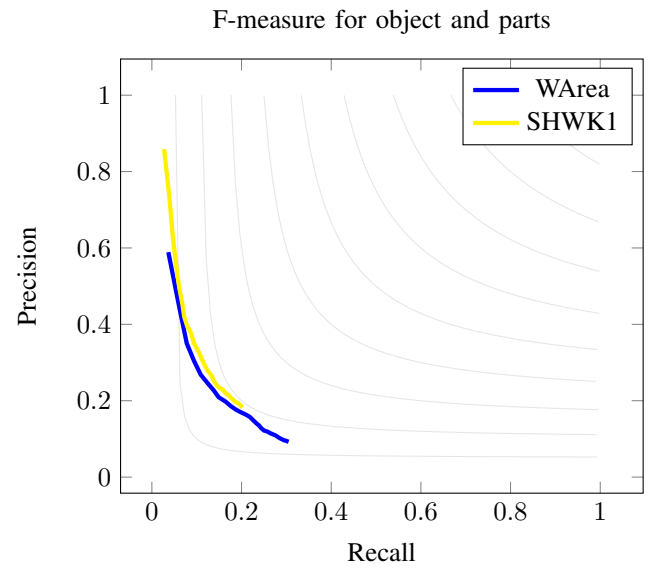

Fig. 10. Comparison using $F_{o p}$.

Enforcing the results of covering, the comparison of object and parts for stochastic hierarchical watershed overcames watershed area in precision, as shown in Fig. 10. In this figure, we compare the hierarchical watershed area (WArea) to the best fitness experiment of this article ( $S H W K 1)$.

\section{CONCLUSION}

This article presented a hierarchical stochastic image segmentation approach which adds uniform random noise to the topographic surface for watershed cuts segmentation. Several segmentations are taken from different noised topographic surfaces of the same image to generate at the end, the final segmentation. The proposed approach is then compared to watershed cuts based on area operator. This one is used as 
baseline because according to literature this measure of image segments presents the best results for hierarchical watershed cuts. Although other measures like volume are also good, area is a very simple one.

In terms of ground-truth covering and F-Measure for object and parts for few number of segments, the noise addition outperfoms the watershed area. Further, the use of a Gaussian convolution using kernel $K 1$ on the averaged saliency map improves the results for the $S H W$.

About noise, just a very small noise energy is enough. Experiments show that increasing the noise energy degrades the ground-truth covering measurement due to the corruption caused on the topographical surface. Further, literature says that noise must be no stronger than the inherent noise of the input image. Because artificial noise should ideally not be stronger than the noise in the input image, as a future work, image noise estimations [31] could be used to set this parameter.

\section{ACKNOWLEDGMENT}

The authors are grateful to CAPES/COFECUB (592/08), CAPES/PVE (88881.064965/2014-01), FAPEMIG (PPM-00616 and PPM-177-14), CNPq and PUC Minas for the financial support to this work.

\section{REFERENCES}

[1] M. Eitz, J. Hays, and M. Alexa, "How do humans sketch objects?" ACM Trans. Graph., vol. 31, no. 4, pp. 44:1-44:10, Jul. 2012.

[2] S. Beucher and C. Lantuéjoul, "Use of watersheds in contour detection," workshop published, Sep. 1979.

[3] L. Vincent and P. Soille, "Watersheds in digital spaces: An efficient algorithm based on immersion simulations," IEEE Trans. Pattern Anal. Mach. Intell., vol. 13, no. 6, pp. 583-598, Jun. 1991.

[4] F. Meyer, "Topographic distance and watershed lines," Signal Process., vol. 38, no. 1, pp. 113-125, Jul. 1994.

[5] L. Najman and M. Schmitt, "Watershed of a Continuous Function," Signal Processing, vol. 38, no. 1, pp. 99-112, 1994, special issue on Mathematical Morphology.

[6] M. Couprie and G. Bertrand, "Topological grayscale watershed transformation," in IN SPIE VISION GEOMETRY V PROCEEDINGS, 3168 1997, pp. 136-146.

[7] A. Bieniek, A. Moga, A. ludwigs-universitat Freiburg, P. D. ing, and H. Burkhardt, "A connected component approach to the watershed segmentation," in In Mathematical Morphology and its Applications to Image and Signal Processing. Springer, 1998, pp. 215-222.

[8] S. Beucher and F. Meyer, "The morphological approach to segmentation: the watershed transformation. Mathematical morphology in image processing." Optical Engineering, vol. 34, pp. 433-481, 1993.

[9] G. Bertrand, "On topological watersheds," Journal of Mathematical Imaging and Vision, vol. 22, no. 2, pp. 217-230, 2005.

[10] M. Couprie, L. Najman, and G. Bertrand, "Quasi-linear algorithms for the topological watershed," Journal of Mathematical Imaging and Vision, vol. 22, no. 2-3, pp. 231-249, May 2005.

[11] L. Najman, M. Couprie, and G. Bertrand, "Watersheds, mosaics and the emergence paradigm," Discrete Applied Mathematics, vol. 147, no. 2-3, pp. 301-324, Apr. 2005, special issue on Discrete Geometry for Computer Imagery.

[12] S. Beucher, "Watershed, hierarchical segmentation and waterfall algorithm," in Mathematical Morphology And Its Applications To Image Processing, ser. Computational Imaging And Vision, Serra, J and Soille, P, Ed., vol. 2, 1994, Proceedings Paper, pp. 69-76.

[13] L. Najman and M. Schmitt, "Geodesic saliency of watershed contours and hierarchical segmentation." IEEE Trans. Pattern Anal. Mach. Intell., vol. 18 , no. 12 , pp. 1163-1173, 1996.
[14] P. Arbelaez, M. Maire, C. Fowlkes, and J. Malik, "Contour detection and hierarchical image segmentation," IEEE Trans. Pattern Anal. Mach. Intell., vol. 33, no. 5, pp. 898-916, May 2011.

[15] J. Cousty and L. Najman, "Incremental algorithm for hierarchical minimum spanning forests and saliency of watershed cuts," in 10th International Symposium on Mathematical Morphology (ISMM'11), ser. Lecture Notes in Computer Science, vol. 6671, Verbania-Intra, Italy, Jul. 2011, pp. 272-283.

[16] D. Martin, C. Fowlkes, D. Tal, and J. Malik, "A database of human segmented natural images and its application to evaluating segmentation algorithms and measuring ecological statistics," in Proc. 8th Int'l Conf. Computer Vision, vol. 2, July 2001, pp. 416-423.

[17] J. Angulo and D. Jeulin, "Stochastic watershed segmentation," Proc. of the 8th International Symposium on Mathematical Morphology, pp. 265-276, 2007.

[18] J. Angulo, S. Velasco-Forero, and J. Chanussot, "Multiscale stochastic watershed for unsupervised hyperspectral image segmentation," in IEEE International Geoscience \& Remote Sensing Symposium, IGARSS 2009, July 12-17, 2009, University of Cape Town, Cape Town, South Africa, Proceedings, 2009, pp. 93-96.

[19] G. Noyel, J. Angulo, and D. Jeulin, "Classification-driven stochastic watershed. application to multispectral segmentation," in 4th European Conference on Colour in Graphics, Imaging, and Vision and 10th International Symposium on Multispectral Colour Science, CGIV 2008/MCS'08, Terrassa, Spain, June 9-13, 2008, 2008, pp. 471-476.

[20] K. B. Bernander, K. Gustavsson, B. Selig, I.-M. Sintorn, and C. L. Luengo Hendriks, "Improving the stochastic watershed," Pattern Recognition Letters, vol. 34, no. 9, pp. 993-1000, Jul. 2013.

[21] F. Malmberg, B. Selig, and C. L. Luengo Hendriks, "Exact evaluation of stochastic watersheds: From trees to general graphs," in Discrete Geometry for Computer Imagery, ser. Lecture Notes in Computer Science, E. Barcucci, A. Frosini, and S. Rinaldi, Eds., vol. 8668. Springer, 2014, pp. 309-319.

[22] F. Meyer and J. Stawiaski, "A stochastic evaluation of the contour strength." in DAGM-Symposium, ser. Lecture Notes in Computer Science, M. Goesele, S. Roth, A. Kuijper, B. Schiele, and K. Schindler, Eds., vol. 6376. Springer, 2010, pp. 513-522.

[23] L. Najman and M. Schmitt, "A dynamic hierarchical segmentation algorithm," in Mathematical Morphology and its applications to Signal Processing II, no. 1, Fontainebleau, France, France, 1994, p. 10pp., poster session.

[24] J. Cousty, G. Bertrand, L. Najman, and M. Couprie, "Watershed cuts: Minimum spanning forests and the drop of water principle," IEEE TRANS. PATTERN ANALYSIS AND MACHINE INTELLIGENCE, pp. 1362-1374, 2009.

[25] F. Meyer, "Minimum spanning forests for morphological segmentation," in Proceedings of the 2nd International Symposium on Mathematical Morphology and Its Applications to Image Processing, ISMM 1994, Fontainebleau, France, Septemcurstyber 1994, 1994, pp. 77-84.

[26] L. Najman, J. Cousty, and B. Perret, "Playing with Kruskal: algorithms for morphological trees in edge-weighted graphs," in International Symposium on Mathematical Morphology, ser. Lecture Notes in Computer Science, R. S. C.L. Luengo Hendriks, G. Borgefors, Ed., vol. 7883. Uppsala, Sweden: Springer, May 2013, pp. 135-146.

[27] F. Meyer, "The dynamics of minima and contours," in Proceedings of the 3th International Symposium on Mathematical Morphology and its Applications to Image and Signal Processing, ISMM 1996, Atlanta, GA, USA, May 1996, 1996, pp. 329-336.

[28] B. Perret, J. Cousty, J. C. Rivera Ura, and S. J. F. GuimarÃ£es, "Evaluation of morphological hierarchies for supervised segmentation," in Mathematical Morphology and Its Applications to Signal and Image Processing, ser. Lecture Notes in Computer Science, J. Benediktsson, J. Chanussot, L. Najman, and H. Talbot, Eds., vol. 9082. Reykjavik, Iceland: Springer, May 2015, pp. 39-50.

[29] C. Vachier, "Extraction de caractéristiques, segmentation d'image et morphologie mathématique," Theses, École Nationale Supérieure des Mines de Paris, Dec. 1995

[30] J. Pont-Tuset and F. Marqués, "Measures and meta-measures for the supervised evaluation of image segmentation," in Computer Vision and Pattern Recognition (CVPR), 06/2013 2013.

[31] J. Immerkaer, "Fast noise variance estimation," Computer Vision and Image Understanding, vol. 64, no. 2, pp. 300 - 302, 1996. 